\title{
Microstructure and Optical Properties of Composites Consisting of Nanoporous Stretched Polypropylene doped with Liquid Crystals and Quantum Dots at a High Concentration
}

\author{
KONSTANTIN MOCHALOV ${ }^{1,2}$, ALEXEY BOBROVSKY ${ }^{3}$, DARIA SOLOVYEVA ${ }^{1,2}$, \\ PAVEL SAMOKHVALOV ${ }^{2}$, IGOR NABIEV ${ }^{2,4}$ and VLADIMIR OLEINIKOV ${ }^{1,2}$
}

${ }^{1}$ Laboratory of Molecular Biophysics, Shemyakin-Ovchinnikov Institute of Bioorganic Chemistry, Russian; Academy of Sciences, Ulitsa Miklukho-Maklaya 16/10, Moscow, 117997 Russian Federation.

${ }^{2}$ Laboratory of Nano-Bioengineering, National Research Nuclear University MEPhl

(Moscow Engineering Physics Institute), Kashirskoe sh. 31, Moscow, 115409 Russian Federation.

${ }^{3}$ School of Chemistry, Moscow State University, Leninskie gory, Moscow, 119991 Russian Federation.

${ }^{4}$ Laboratoire de Recherche en Nanosciences, LRN-EA4682, UFR de Pharmacie,

Université de Reims Champagne-Ardenne, 51100 Reims, France.

*Corresponding author E-mail: voleinik@mail.ru

http://dx.doi.org/10.13005/ojc/320604

(Received: November 25, 2016; Accepted: December 12, 2016)

\begin{abstract}
Unique properties of nanohybrid composites based on different types of porous polymer matrices doped with fluorescent nanoparticles (quantum dots, QDs) are determined by the combination of the mechanical properties of the host matrix (flexibility, chemical stability, etc.) and a high luminescence intensity and extreme stability of QDs. Here, we report on the preparation, optical and microstructural characterization of a nanoporous stretched polypropylene matrix embedded with CdSe/ZnS QDs as fluorescent dopants at a high concentration. The microstructure and optical properties of two types films based on QD-polymer composites and liquid crystals are described. The distribution of QDs in the composite films and their morphology was determined. The annealing of the nanoporous composite films leads to shrinking of the pores and encapsulation of QDs, which results in a domain-like structure. The resulting flexible, stable and highly luminescent materials may be applied to obtain the highly luminescent diodes, the light converter devices and the display systems.
\end{abstract}

Keywords: quantum dot-polymer composites; nanoporous polypropylene; fluorescence.

\section{INTRODUCTION}

Designing organic-inorganic hybrid polymer composites has been a remarkable challenge in recent decades. Such composites combine good mechanical properties of polymer matrices with unique optical, electrical, and other characteristics of the inorganic dopants ${ }^{1,2}$. Semiconductor fluorescent 
quantum dots (QDs) are among the most prominent fluorescent dopants, attracting increasing attention due to their unique optical properties. QDs have broad light absorption bands and intense, narrow fluorescence bands whose spectral positions vary from the IR to almost the UV region. The wavelengths of the absorbance and fluorescence maximums can be easily tuned by varying the QD size, which is determined by the conditions of their synthesis ${ }^{3,4}$. These properties of QDs make them the most promising type of fluorescent dopants for the development of nanohybrid composites based on different types of polymer matrices. Among the main lines of research in this field, noteworthy are the development of techniques for highly homogeneous aligned distribution of QDs in different organic matrices ${ }^{5-9}$, designing of light conversion devices ${ }^{10-}$ ${ }^{12}$, and sensing approaches ${ }^{13,14}$. Significant efforts have been made to engineer hybrid nanophotonic materials from different types of liquid crystals and QDs ${ }^{15,16}$. Our research group has taken part in the development of photonic devices with photo- and electrically controlled fluorescent properties based on cholesteric liquid crystal matrices doped with CdSe/ZnS semiconductor fluorescent QDs ${ }^{17-19}$, as well as the development of the techniques for characterization of polymer matrix-QD hybrid systems ${ }^{20}$. The main difficulty with most of the polymer matrix doping processes is an extremely low concentration of the homogeneously dispersed QDs, which prevents the use of these systems for engineering of highly luminescent devices.

In order to overcome this problem, we employed a new strategy based on encapsulation of QDs into pores of porous polymers. In our previous studies ${ }^{21,22}$, a simple and robust method of preparation of QD-polymer composite films out of nanoporous polypropylene (PP) or polyethylene $(\mathrm{PE})$ and CdSe/ZnS QDs was elaborated. In the study ${ }^{16}$, two types of PP-based composite films were prepared. The first type was obtained by absorption of CdSe/ZnS QDs onto the porous structure of PP films, followed by annealing above the melting point of PP. The second type of composite films was obtained by filling porous QD-PP composites with a nematic liquid crystal (LC) mixture. It has been shown that both types of composites are characterized by low light scattering, which makes it possible to study their optical properties by means of absorbance and fluorescence spectroscopies.

The present paper describes detailed investigation of the microstructure and optical properties of such QD-polymer-liquid crystal (QDPP-LC) composite films. For this purpose, two series of samples were prepared. One of them (Sample 1) was obtained by filling PP with liquid crystals and a concentrated solution of QDs in octadecene; the other (Sample 2), by filling PP with liquid crystals and a QD solution in octadecene followed by washing the film with acetone and annealing it at $170^{\circ} \mathrm{C}$.

Octadecene is a good solvent for QDs, with a very high boiling point $\left(315^{\circ} \mathrm{C}\right)$ and a refractive index close to that of PP. The similarity of refractive indices allow transparent composite films to be obtained; the high boiling temperature and low vapor pressure ensure their stability.

The main goal of this study was detailed analysis of the nanoporous structure and fluorescent properties of the obtained composite films using optical and atomic force microscopies and confocal fluorescence microspectroscopy. This combination of microscopy methods allowed us to obtain data on the morphology of composite films and QD distribution in them. Proposed approach enable controlled engineering of nanohybrid composites with desired morphology and physical, mechanical and optical properties.

\section{EXPERIMENTAL}

\section{Nanoporous PP films}

Nanoporous PP films were purchased from Celgard (a CelgardR 2500 microporous monolayer membrane). The thickness of the films was $25 \mathrm{im}$, the porosity was $55 \%$, and the average pore size was $0.064 \mu \mathrm{m}$.

\section{Synthesis of QDs}

The procedure of the synthesis of CdSe/ ZnS core/shell QDs is described in detail in ${ }^{3}$. CdSe cores with a diameter of $\sim 2.9 \mathrm{~nm}$ were synthesized by the hot-injection method, using $n$-hexadecylphosphonic acid as a capping agent. After isolation and purification, the CdSe cores 
were coated with a three-monolayer $\mathrm{ZnS}$ shell in an octadecene-oleylamine solution, after which the native surface capping ligands were replaced with tri- $n$-octyl phosphine oxide (TOPO). The fluorescence quantum yield of the core/shell QDs was $68 \%$, as estimated using rhodamine $6 G$ as a reference dye. Prior to introducing them into the PP matrix, the purified CdSe/ZnS QDs were dissolved in 1 -octadecene $(20 \mathrm{mg} / \mathrm{mL})$.

\section{Preparation of QD-polymer-liquid crystal composites}

The QD-PP-LC composites (Sample 1) were prepared by coating nanoporous PP films with a concentrated QD solution in octadecene (20 $\mathrm{mg} / \mathrm{mL}$ ). After complete filling of the films with the solutions, which took a few seconds, excess solution was removed using filter paper. Then, a LC based on MLC6816 (cyclohexane derivatives, Merck) was introduced into the porous structure of the PP-QD films.

Sample 2 was prepared by filling PP with the same $Q D$ solution and $L C$ in octadecene followed by washing the film with acetone and annealing it at $170^{\circ} \mathrm{C}$.

\section{Methods}

Fluorescence confocal images were in the spectral range of $583-592 \mathrm{~nm}$ for all samples. The fluorescence spectra were recorded using an M266 automated monochromator/spectrograph (SOLAR Laser Systems) with an U2C-16H7317 CCD (Ormins) and a homemade light-collecting inverted system using a 100X/0.80 MPLAPON lens (Olympus) and a homemade confocal unit with two $100-\mathrm{mm}$ objective lenses, a $100-\mu \mathrm{m}$ pinhole, and two Semrock 488-nm RazorEdge ${ }^{\circledR}$ ultrasteep long-pass edge filters (Semrock). An LGN-519M $\mathrm{Ar}^{+}$laser (Plazma) operating at $488 \mathrm{~nm}$ was used for fluorescence excitation at a light intensity of 0.1 $\mathrm{mW}$, as measured with a LaserMate-Q (Coherent) intensity meter. An integration time of $0.03 \mathrm{~s}$ was used for recording confocal images. The scan area was $10 \times 10 \mu \mathrm{m}(100 \times 100$ points $)$.

AFM and upright microscopy images were acquired using an SPM Nntegra base (NTMDT) equipped with an Optem Zoom 125C upright microscope (Qioptiq). This SPM base was combined with the aforementioned inverted microscopic system, allowing us to obtain AFM, inverted microscopic, and upright microscopic images from the same area of the sample. For AFM images, a scan area of $8 \times 8 \mu \mathrm{m}(512 \times 512$ points $)$ and AFM cantilevers of the ETALON HAFM series (NT-MDT) were used.

\section{RESULTS AND DISCUSSION}

Figure 1 shows the absorbance and fluorescence spectra of the obtained samples. As seen from the figure, both samples exhibit the light absorption and emission characteristics determined by the high concentration of QDs. The fluorescence maximum for Sample $2(589 \mathrm{~nm})$ is red-shifted relative to that of Sample1 $(585 \mathrm{~nm})$, probably, due to energy transfer.

The upright and inverted optical images of Sample 1 (Figures 2a, 2b) and Sample 2 (Figures 2c, 2d) are shown in Figure 2. The most distinctive feature of the two samples is a homogeneous opaque surface, which strongly scatters light in the upright mode in the unannealed sample (Figure 2a). The Sample 2 surface has a domainlike structure formed by tightly packed optically transparent "domains" separated by opaque borders and is also well observed in the upright mode (Figure 2c).

Both samples are similarly and intensely colored due to the embedded QDs, the coloration being apparently uniform. They, however, significantly differ in transparency. The Sample 1 optical image obtained in the inverted mode (Figure 2b) is mostly a continuous dark field.

Sample 2 is completely transparent at the same light intensity (Figure 2d). In this case, the inner radial structure of the domains converging to the center and the domain boundaries are clearly visible. However, the defective, in terms of light scattering, areas of Sample 1 deserve special attention. They are seen as dark spots in Figure 2a (area 1). A surprising fact is that these spots are the only light (transparent) areas in the optical images obtained in the inverted mode. The inverted image of area 1 (Figure 2a) is shown in Figure 2b. The nature of the transparent areas in Sample 1 (PP CelgardR 2500) will be studied further, but at this point it is important 
to note that the reference sample is not completely transparent and exhibits a very high light scattering with no localized areas of transparency.

The next step in investigating the properties of the materials obtained (PP CelgardR 2500/ $\mathrm{QD)}$ was to study the surface topography of these samples by means of AFM. The AFM images of the reference sample PP CelgardR 2500 (Figure 3a),
Sample 1 (Figures 3c, 3d), and Sample 2 (Figure 3e) and the corresponding cross sections are shown in Figure 3. The AFM scanning direction of all the samples coincided with the direction of the polypropylene matrix tension. The result of this location of the sample is the structure with an undulating "large" period (on average, about 1 im) related to an almost uniform rupture of the polypropylene matrix along the tension direction
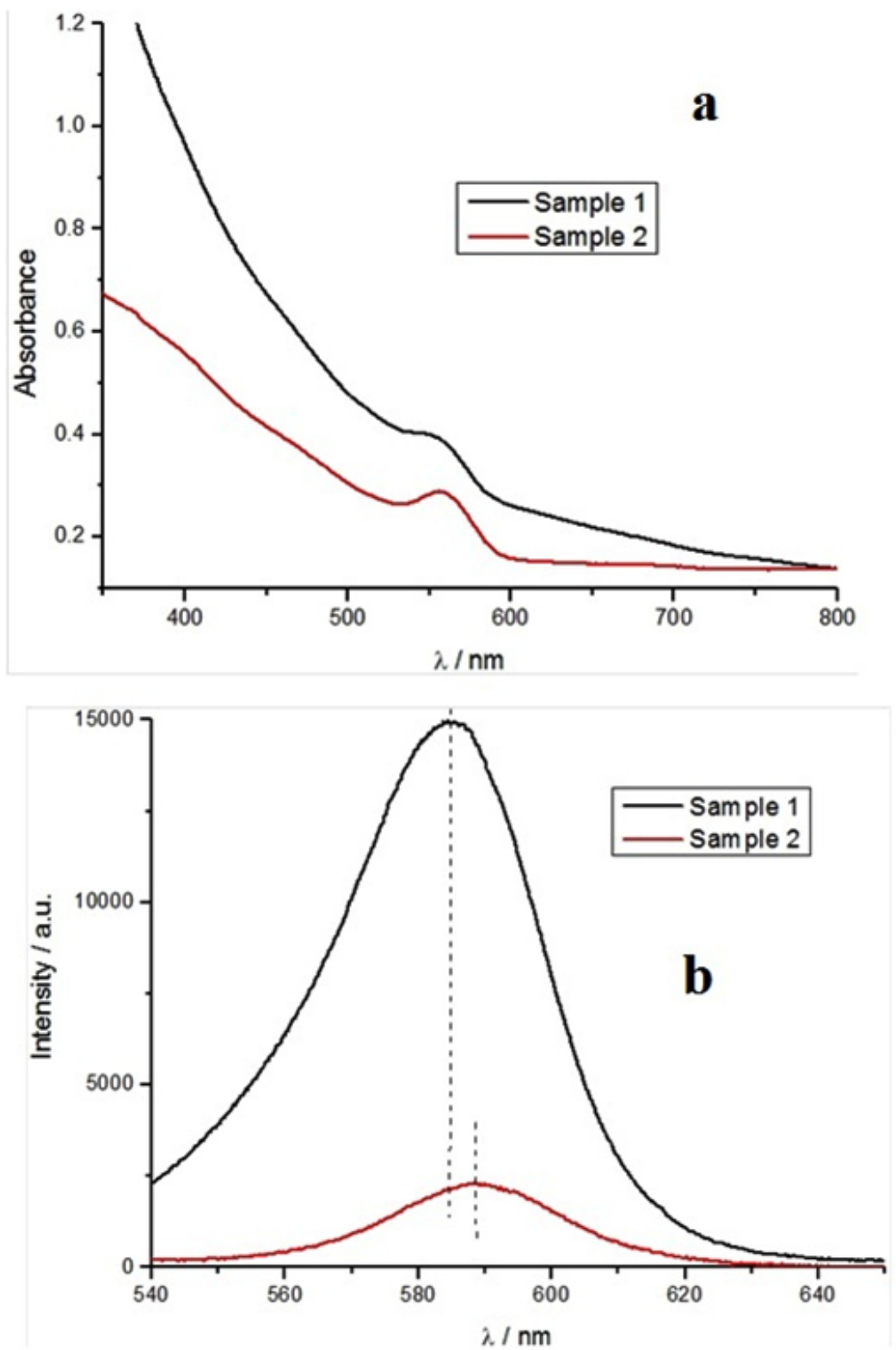

Fig. 1: The (a) absorbance and (b) fluorescence spectra of two samples; The fluorescence excitation wavelength is $532 \mathrm{~nm}$ 
in all AFM images. Thus, relatively long (up to tens of micrometers) filamentary structures about $100 \mathrm{~nm}$ in height and as wide as several hundred nanometers perpendicular to the scanning direction are formed (Figures 3a, 3b, upper panel). There are also filamentary structures with a "small" period $(100 \mathrm{~nm})$ located almost perpendicularly to those "large" structures. The "small" filamentary structures are obviously formed by the polypropylene matrix fragments remaining after spatial separation of the "large" filamentary structures (Figures 3a, 3b, bottom panel). Transverse parameters of the "small" filaments cannot be accurately measured by AFM, since the aspect ratio of the cantilever tip does not allow it to penetrate between them. However, we can assume that the samples have about the same 3D network-like structure consisting of "large" and "small" in their bulk. The average porosity can be estimated at about $60 \%$, assuming the average ratio of the "large" filament width to the distance between the filaments to be $1 / 3$ and the same parameter for the "small" filaments to be 1/2. The porosity estimated using conventional techniques is close to $55 \%{ }^{23}$. Good agreement of these values means that the bulk structure of Sample 1 generally corresponds to the surface morphology revealed with AFM and consists of mutually perpendicular "small" and "large" filamentary structures. This porosity value
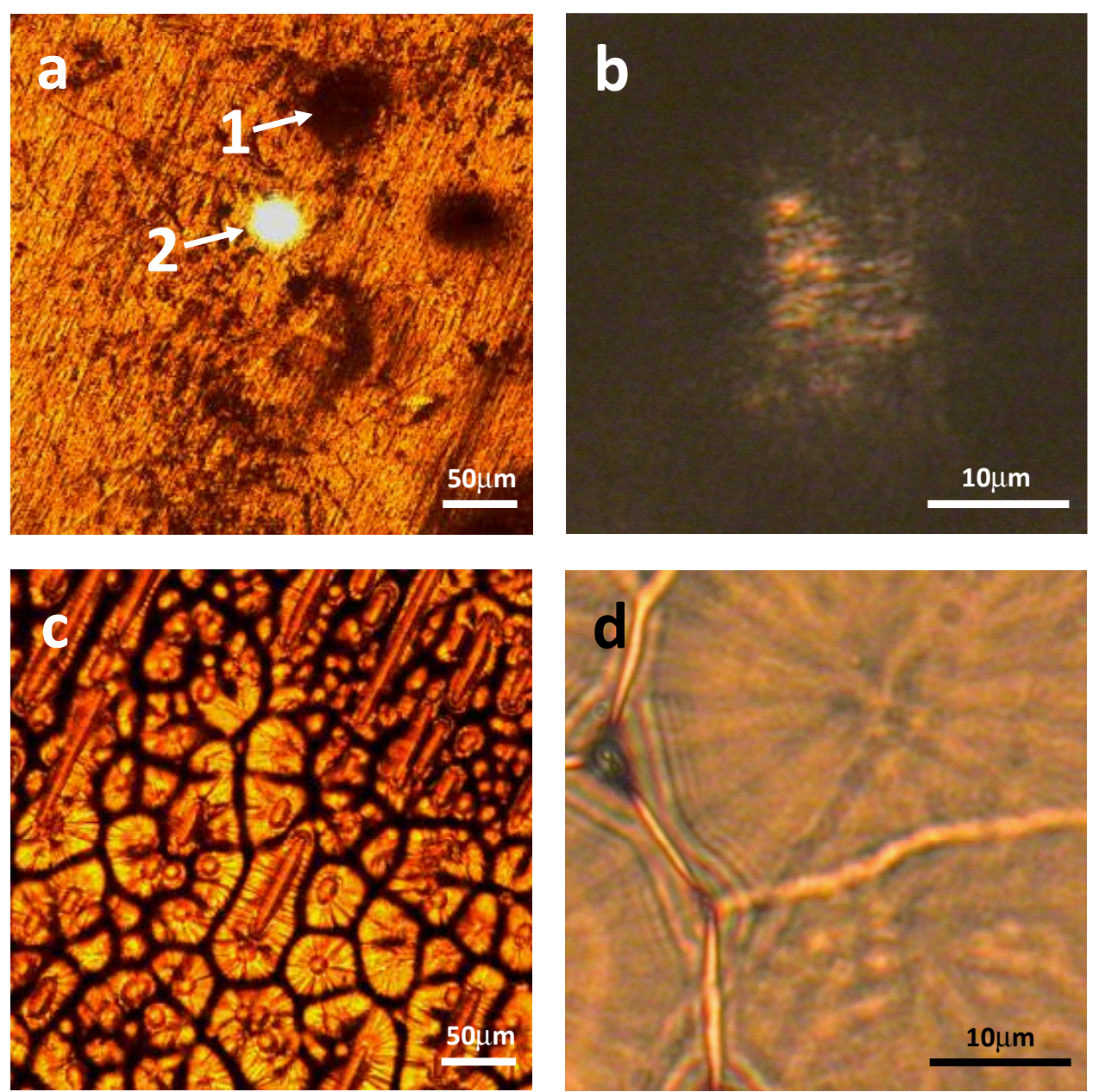

Fig. 2: Upright $(a, c)$ and inverted (b, d) light microscopy images of Sample $1(a, b)$ and Sample 2 (c, d). Dark round area 1 in the upright image (panel a) is the same that in the inverted image (panel b). Bright spot 2 in the upright image (panel a) is the fluorescence excitation laser spot 
indicates the possibility of introducing a substantial amount of a dopant (in the given case, QDs) into the material.
Analysis of the morphology of QD distribution in the matrix was the next step of this study. A large-scale view of the Sample 1 surface is
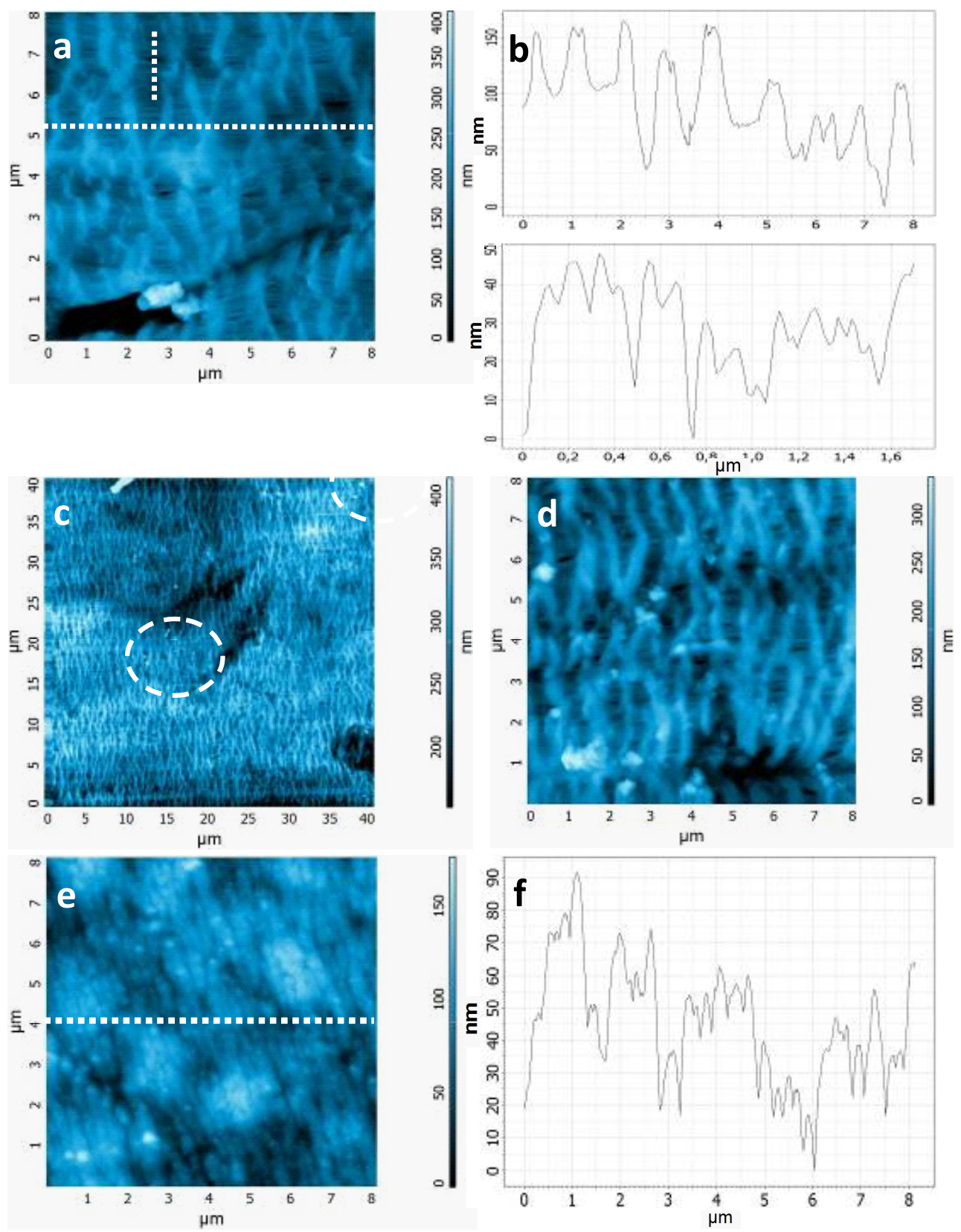

Fig. 3: (a) An AFM image of the reference PP CelgardR 2500 sample. The white dotted lines indicate cross sections. A typical but rare defect of this structure is seen in the left bottom corner. (b) The profiles of the horizontal (top) and vertical (bottom) cross sections shown in panel a. (c) Large-scale and (d) closeup AFM images of the Sample 1 area enriched with QDs (marked with a white dashed circle in panel c). (e) An AFM image of Sample 2 and (f) the profile of its cross section indicated by the white dotted line 
shown in Figure 3c. As seen from the figure, QDs are not distributed uniformly throughout the sample surface; instead, they form islands (marked with dashed circles in Figure 3c). Comparison of AFM images and optical images obtained in the upright mode shows that all regions of QD accumulation in AFM images coincide with dark spots in optical images obtained in the upright mode, such as those shown in Figure 2a (area 1) and areas of local transparency in Sample 1 shown in Figure 2b. The AFM image of area 1 (Figure 2a) of the Sample 1 surface is shown in Figure $3 d$. Here, QDs form submicrometer- and micrometer-sized agglomerates. Probably, the formation of these large QD agglomerates contributes to the substantial change in the scattering properties of the QD-PP-
LC material without altering the overall PP matrix morphology.

Annealing of these samples led to their contraction, with "large" filamentary structures moving close to one another up to a complete absence of intervals between them, while the filament sizes remained unchanged (Sample 2; Figures $3 e, 3 f)$. The surface structure of Sample 2 did not allow us to use AFM for determining the location of QDs on its surface, but it did not detect any local area of QD accumulation, which agrees with the absence of the corresponding defects in optical images of Sample 2 (Figure 2). Evidently, the contraction of the structure as a result of annealing contributes to the effect of the QD "locking" in the
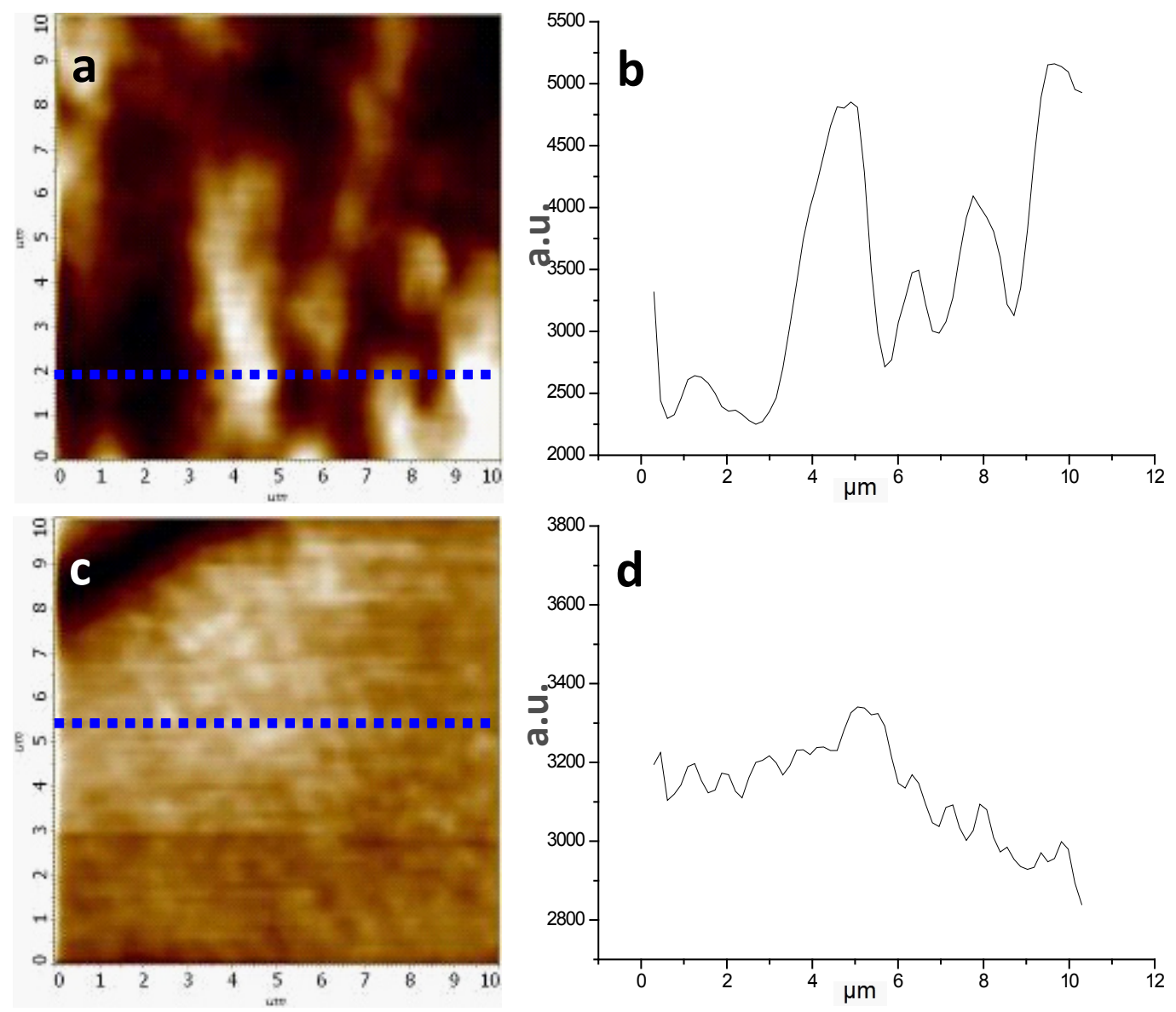

Fig. 4: Confocal fluorescence images of (a) Sample 1 and (c) Sample 2. The distributions of fluorescence intensity along the cross sections of (b) Sample 1 and (d) Sample 2 indicated by the blue dotted lines in panels a and c, respectively 
PP matrix, which results in a flexible material with highly stable fluorescent characteristics.

The final step of this study was to evaluate the homogeneity of the distribution of QDs embedded in the LC and PP matrix, as well as the effects of the washing and annealing procedures on this characteristic. Confocal fluorescence microspectroscopy was used for this purpose. Confocal fluorescent images of Sample 1 and Sample 2 are shown in Figures $4 \mathrm{a}$ and 4c, respectively.

The fluorescence profiles of cross sections indicated by the blue dotted lines in the confocal images (Figures 4a, 4c) reflect the differences in the morphology of QD distribution in Samples 1 and 2 (Figures $4 b$ and $4 d$, respectively). As seen from Figures $4 \mathrm{~b}$ and $4 \mathrm{~d}$, the annealing (Sample 2) made the QD distribution in the PP matrix substantially more uniform. The total difference in fluorescence intensity within each "domain" of the structure did not exceed 10\% (Figure 4d.), with QDs almost entirely absent at the domain boundaries (the upper left corner of the image in Figure 4c). Before annealing (Sample 1), the QD distribution was substantially less homogeneous. The total difference in fluorescence intensity (more than 60\%) was accounted for by numerous local inhomogeneities (Figure 4b). The locations of these inhomogeneities mainly coincided with the locations of dark spots in the optical images obtained in the upright mode. For example, in area 1 (Figure 2a), the QD fluorescence intensity was three times higher than that in area 2 (laser spot showed) with a relatively homogeneous QD distribution. It should be noted that the highly heterogeneous distribution of QD fluorescence in Sample 1 (Figure $4 b$ ) is also observed in the areas that look homogeneous under an upright optical microscope.

Thus, annealing plays an important role in the preparation of PP-QD materials, "locking" QDs in the PP matrix and substantially increasing the homogeneity of their distribution.

\section{CONCLUSIONS}

A new type of nanohybrid materials engineered by encapsulating fluorescent semiconductor QDs into the CelgardR 2500 polypropylene matrix has been characterized with the use of AFM and confocal microspectroscopy. It has been found that stretching of the initial polypropylene matrix resulted in the formation of mutually perpendicular "small" and "large" filamentary structures. The "large" filaments with an average period of about 1 ìm are almost perpendicular to the stretching direction, whereas the "small" filaments (with an average period of about $100 \mathrm{~nm}$ ) are oriented in an almost opposite direction. Comparison of the values of bulk porosity estimated by means of the standard technique and surface morphology analysis suggests that this filamentary structure is characteristic of the bulk PP-QD-LC material. Such a high porosity allows incorporating significant amounts of QDs into the PP matrix. It has been found that annealing of the PP-QD-LC nanohybrid material leads to shrinking of the pores formed with crossed filaments and, as a result, encapsulation of the incorporated QDs and formation of a domain-like structure. Moreover, it has been shown that annealing significantly (sixfold) increases the homogeneity of the QD distribution within each domain.

In conclusion, it should be noted that encapsulation of fluorescent semiconductor QDs into an annealed PP matrix yields a flexible and stable material characterized by highly intense and homogeneous fluorescence. The most appropriate areas of application of such hybrid systems are the development of sensors systems ${ }^{24-26}$, highly luminiscent diodes, including white light-emitting ones $^{11,27,28}$, light converter devices ${ }^{12}$, and display systems ${ }^{29}$.

\section{ACKNOWLEDGEMENTS}

This study was supported by the Ministry of Higher Education and Science of the Russian Federation (grant no. 14.616.21.0042 ID RFMEFI61615X0042.) 


\section{REFERENCES}

1. Lvov, Yu.; Guo, B.; Fakhrullin, R. F. (Eds.). Functional Polymer Composites with Nanoclays. RSC Publishing, 2016.

2. Mark, J. E.; Lee, C. Y-C.; Bianconi, P. A. (Eds.). Hybrid Organic-Inorganic Composites. American Chemical Society, 1995. DOI: 10.1021/bk-1995-0585.fw001

3. Rogach, A. Semiconductor Nanocrystal Quantum Dots: Synthesis, Assembly, Spectroscopy and Applications. Berlin: Springer, 2008.

4. Sukhanova, A.; Venteo, L.; Devy, J.; Artemyev, M.; Oleinikov, V.; Pluot, M.; Nabiev, I. Highly Stable Fluorescent Nanocrystals as a Novel Class of Labels for Immunohistochemical Analysis of Paraffin-Embedded Tissue Sections. Lab. Invest. Brief Meth., 2002, 82, 1259-1261.

5. Gugula, K.; Szydlo A.; Stegemann, L.; Strassert, C. A.; Bredol, M. Photobleachingresistant ternary quantum dots embedded in a polymer-coated silica matrix. J. Mater. Chem. C., 2016, 4, 5263-5269.

6. Thorkelsson, M.; Nelson, J. H.; Alivisatos, A. P.; Xu, T. End-to-End Alignment of Nanorods in Thin Films. Nano Lett., 2013, 13, 49084913.

7. Matvienko, O. O.; Savin, Y. N.; Kryzhanovska, A. S.; Vovk, O. M.; Dobrotvorska, M. V.; Pogorelova, N. V.; Vashchenko, V. V. Dispersion and aggregation of quantum dots in polymerinorganic hybrid films. Thin Solid Films, 2013, 537, 226-230.

8. Rünzi, T.; Baier, M.C.; Negele, C.; Krumova, M.; Mecking, S. Nanocomposites of phosphonicacid-functionalized polyethylenes with inorganic quantum dots. Macromol. Rapid. Commun., 2015, 36, 165-173.

9. Krini, R.; Ha, C. W.; Prabhakaran, P.; Mard, H. E.; Yang, D.-Y.; Zentel, R.; Lee, K.-S. Photosensitive functionalized surface-modified quantum dots for polymeric structures via twophoton-initiated polymerization technique. Macromol. Rapid Commun., 2015, 36, 11081114.

10. Meinardi, F.; Colombo, A.; Velizhanin, K. A.; Simonutti, R.; Lorenzon, M.; Beverina, L.; Viswanatha, R.; Klimov, V. I.; Brovelli, S. Large area luminescent solar concentrators based on "Stokes-shift-engineered" nanocrystals in mass polymerized polymethylmethacrylate matrix. Nature Photonics, 2014, 8, 392-399.

11. Kahmann, S.; Mura, A.; Protesescu, L.; Kovalenko, M. V.; Brabec, C. J.; Loi, M. A. Optoelectronics of $\mathrm{PbS}$ quantum dot and narrow bandgap polymer blends. J. Mater. Chem. C., 2015, 3, 5499-5505.

12. Chen, C.-J.; Lin, C.-C.; Lien, J.-Y.; Wang, S.-L.; Chiang, R.-K. Preparation of quantum dot/ polymer light conversion films with alleviated Förster resonance energy transfer redshift. $J$. Mater. Chem. C., 2015, 3, 196-203.

13. He, X.; Tan, L.; Wu, X.; Yan, C.; Chen, D.; Meng, X.; Tang, F. Electrospun quantum dots/polymer composite porous fibers for turn-on fluorescent detection of lactate dehydrogenase. J. Mater. Chem., 2012, 22, 18471-18478.

14. Generalova, A. N.; Oleinikov, V. A.; Sukhanova, A.; Artemyev, M. V.; Zubov, V. P.; Nabiev, I. Quantum dot-containing polymer particles with thermosensitive fluorescence, Biosensor and Bioelectronics. Biosens. Bioelectron., 2013, 39, 187-193.

15. Tong, X.; Zhao, Y. Liquid-Crystal Gel-Dispersed Quantum Dots: Reversible Modulation of Photoluminescence Intensity Using an Electric Field. J. Am. Chem. Soc., 2007, 129, 6372-6373.

16. Mirzaei, J.; Reznikov, M.; Hegmann, T. Quantum dots as liquid crystal dopants. J. Mater. Chem., 2012, 22, 22350-22365.

17. Bobrovsky, A.; Mochalov, K.; Oleinikov, V.; Shibaev, V. Glass-forming photoactive cholesteric oligomers doped with quantum dots: novel materials with phototunable circularly polarised emission. Liq. Cryst., 2011, 38, 737-742.

18. Bobrovsky, A.; Mochalov, K.; Oleinikov, K.; Sukhanova, A.; Prudnikau, A.; Artemyev, M.; Shibaev, V.; Nabiev, I. Optically and electrically controlled circularly polarized Emission from cholesteric liquid crystal materials doped with semiconductor quantum dots. Adv. Mater., 2012, 24, 6216-6222.

19. Bobrovsky, A.; Samokhvalov, P.; Shibaev, V. 
An effective method for preparation of stable LC composites with high concentration of quantum dots. Advanced Optical Materials, 2014, 2, 1167-1172.

20. Mochalov, K.E.; Efimov, A.E.; Bobrovsky, A.; Agapov, I.I.; Chistyakov, I.I.; Oleinikov, V.; Sukhanova, A.; Nabiev, I. Combined Scanning Probe Nanotomography and Optical Microspectroscopy: A Correlative Technique for 3D Characterization of Nanomaterials. ACS Nano, 2013, 7, 8953-8962.

21. Bobrovsky, A.; Shibaev, V.; Elyashevitch, G.; Mochalov, K.; Oleynikov, V. Polyethylene-based composites containing high concentration of quantum dots. Colloid Polym. Sci., 2015, 293, 1545-1551.

22. Bobrovsky, A.; Shibaev, V.; Abramchuk, S.; Elyashevitch, G.; Samokhvalov, P.; Oleinikov, V.; Mochalov, K. Quantum dotpolymer composites based on nanoporous polypropylene films with different draw ratios. Eur. Polym. J., 2016, 82, 93-101.

23. Celgard product information. Date Views: 14.09.2016 http://www.ldcgm.com/Celgard/ CELGA, RD-4550.pdf

24. Han, S.; Li, X.; Wang, Y.; Sub, C. A core-shell $\mathrm{Fe}_{3} \mathrm{O}_{4}$ nanoparticle-CdTe quantum dotmolecularly imprinted polymer composite for recognition and separation of 4-nonylphenol. Anal. Methods., 2014, 6, 2855-2861.

25. Zhou, Y.; Qu, Z.; Zeng, Y.; Zhou, T.; Shi, G. A novel composite of graphene quantum dots and molecularly imprinted polymer for fluorescent detection of paranitrophenol. Biosensors Bioelectr., 2014, 52, 317-323.

26. Liu, H.; Zhou, K.; Wu, D.; Wang, J.; Sun, B. A novel quantum dots-labeled on the surface of molecularly imprinted polymer for turnoff optosensing of dicyandiamide in dairy products. Biosensors Bioelectr., 2016, 77, 512-517.

27. Min, S.-Y.; Bang, J.; Park, J.; Lee, C.-L.; Lee, S.; Park, J.-J.; Jeong, U.; Kim, S.; Lee, T.-W. Electrospun polymer/quantum dot composite fibers as down conversion phosphor layers for white light emitting diodes. RSC Adv., 2014, 4, 11585-11589.

28. Yang, Z.; Voznyy, O.; Liu, M.; Yuan, M.; Ip, A.H.; Ahmed, O.S.; Levina, L.; Kinge, S.; Hoogland, S.; Sargent, E.H. All-Quantum-Dot Infrared Light-Emitting Diodes. ACS Nano, 2015, 9, 12327-12333.

29. Wood, V.; Panzer, M.J.; Chen, J.; Bradley, M.S.; Halpert, J.E.; Bawendi, M.G.; Bulovic, V. Inkjet Printed Quantum Dot-Polymer Composites for Full Color AC Driven Displays. Adv. Mater., 2009, 21, 2151-2155. 